\title{
USO ANTRÓPICO DE LAGARTOS (TUPINAMBIS SP.) EN EL SITIO BELTRÁN ONOFRE BANEGAS-LAMI HERNÁNDEZ (SANTIAGO DEL ESTERO).
}

\section{ANTHROPIC USE OF LIZARDS (TUPINAMBIS SP.) AT BELTRÁN ONOFRE BANEGAS-LAMI HERNÁNDEZ SITE (SANTIAGO DEL ESTERO PROVINCE).}

\author{
Luis M. del Papa ${ }^{1}$ y Leda Moro \\ 1 CONICET. Facultad de Ciencias Naturales y Museo, Universidad Nacional de La Plata. Calle 64 s/n, entre 120 y \\ diag. 113. E-mail: loesdelpapa@hotmail.com \\ 2 Facultad de Ciencias Naturales y Museo, Universidad Nacional de La Plata. E-mail: ledamoro@gmail.com
}

Presentado el: 19/12/2013 - Aceptado 01/10/2014

\begin{abstract}
Resumen
En los últimos años se han ampliado los análisis arqueofaunísticos hacia la fauna menor. En este caso se analiza los patrones de uso de los lagartos (Tupinambis sp.) en el sitio arqueológico Beltrán Onofre Banegas-Lami Hernández (Dpto. Robles, Santiago del Estero). Este sitio corresponde a un contex to agroalfarero Tardío (con un fechado de $420 \pm 60$ años 14C AP) y se ubica en las inmediaciones de un paleocauce del Río Dulce. Se realizó el análisis tafonómico de la muestra observándose marcas de corte y termoalteración; además, la representación de partes de Tupinambis sp. coincide con la incorporación de las presas en forma entera a los lugares de consumo. Los restos de Tupinambis sp. son recurrentes en los sitios arqueológicos de la región para el contexto agroalfarero, y en algunos casos las evidencias de consumo concuerdan con las presentadas en este trabajo. Si bien los reptiles en la muestra no superan el 6\% del NISP total, los mismos constituyeron un recurso complementario y de importancia en relación al uso estacional de las presas.
\end{abstract}

Palabras claves: Tupinambis sp., Región arqueológica Chaco-Santiagueña, Agroalfarero tardío; Evidencias antrópicas.

\footnotetext{
Abstract

In the last few years, archaeofaunal analysis has extended to small fauna. In this case, the usage patterns of lizards (Tupinambis sp.) at Beltran Onofre Banegas-Lami Hernandez archaeological site have been analyzed (Department of Robles, Santiago del Estero). This site corresponds to a late agro-pottery context (dated to $420 \pm 6014 C$ years BP) and is located in the vicinity of the Rio
} 
Dulce paleobed. A taphonomical analysis of the sample determined cut marks and thermoalteration; in addition, the parts representation of Tupinambis sp. coincides with the introduction of whole preys to the places of consumption. The remains of Tupinambis sp. recur in the archaeological sites of the region for the agro-pottery context, and in some cases consumer evidence is consistent with those described in this paper. While reptiles in the sample do not exceed $6 \%$ of total NISP, they were an important complementary resource in relation to prey's seasonal use.

Key words: Tupinambis sp., Chaco-Santiagueña archaeological region, Late agro-pottery context, Anthropic evidences.

\section{Introducción}

En los últimos años se han ampliado y profundizado los análisis arqueofaunísticos hacia la fauna menor, sin embargo, el aporte sobre las evidencias de consumo de los reptiles son escasos. En este aspecto, se destacan los trabajos que hacen referencia al consumo de saurios del género Tupinambis para tres regiones arqueológicas del país con registros del Holoceno tardío. Los trabajos corresponden a la región Pampeana -sub-área de las sierras de Tandilia (Quintana et al. 2002, 2004), Sierras Centrales de Córdoba (Medina 2008) y Chaco Meridional (Santini 2009).

En este caso se analiza los patrones de uso de los lagartos (Tupinambis sp.) en el sitio arqueológico Beltrán Onofre Banegas-Lami Hernández (Dpto. Robles, Santiago del Estero), ubicado a $27^{\circ} 49^{\prime} 08^{\prime \prime}$ de latitud S y $64^{\circ} 02^{\prime} 43^{\prime \prime}$ de longitud $\mathrm{O}$, correspondiente a la región arqueológica Chaco-Santiagueña del noroeste argentino.

El sitio se integra por su localización en latitud y longitud, al espacio subtropical semiárido y continental. Las temperaturas promedio para la zona de estudio tienen valores de $20^{\circ} \mathrm{C}$, con precipitaciones (se concentran en el período estival) de $550 \mathrm{~mm}$ anuales y capacidad de evapotranspiración potencial mayor, lo que determina una gran deficiencia hídrica (Ledesma 1979). En esta región el reptil más importante desde el punto de vista económico es Tupinambis rufescens (lagarto colorado) con un peso promedio en vida de 4,15 kg. Si bien en la actualidad en la región sólo habita esta especie, según Brizuela (2010), Tupinambis meriane (lagarto overo) podría haber habitado la zona de estudio en una época más húmeda, entre el 1000 y 1260 dC para el sitio arqueológico El Veinte. Debido a que la diferenciación entre ambas especies resulta compleja a través de restos óseos, se decidió asignar los especímenes a nivel de género.

Los lagartos del género Tupinambis, como todos los reptiles, son animales ectotérmicos, por lo que las diferencias térmicas estacionales y diarias condicionan su comportamiento. En este sentido, su actividad es fundamentalmente diurna durante su período trófico (de septiembre hasta abril para la zona de estudio), momento en el que se alimentan en forma diaria, realizan el cortejo, luchas, apareamiento, y donde son susceptibles de ser capturados tanto por humanos como por otros depredadores (Richard 1999). Por el contrario, durante el receso invernal (abril-septiembre) se mantienen en su refugio, donde hibernarán en la estación fría (Porini 2006). Estos lagartos presentan hábitos cavícolas, pueden construir sus propias cuevas o habitar las que abandonan otros animales (e.g., Lagostomus maximus) (Fitzgerald 1992). A su vez, las hembras fecundadas de Tupinambis construyen cuevas en forma de túnel para depositar sus huevos, dichas cuevas tienen una dimensión de $1 \mathrm{~m}$ de longitud y $50 \mathrm{~cm}$ de profundidad. 


\section{Sitio Beltrán Onofre Banegas- Lami Hernández. Contexto cultural}

El sitio se distingue por la presencia de montículos que interrumpen el paisaje en la proximidad de un paleocauce del Río Dulce (Figura 1). Se recuperaron en los trabajos de campo material cerámico que por la observación preliminar de los mismos, se asignan al contexto Sunchitúyoj (agroalfarero Tardío), estando todavía bajo estudio. Se obtuvo un fechado radiocarbónico a partir de muestras de carbón vegetal, de $420 \pm 60$ años ${ }^{14} \mathrm{C} \mathrm{AP} \mathrm{(LP-2054).}$ En este trabajo se tienen en cuenta los materiales rescatados en cinco cuadrículas realizadas durante las campañas de 2008. Las cuadrículas fueron excavadas hasta una profundidad entre los 70 y $80 \mathrm{~cm}$ por niveles artificiales de $10 \mathrm{~cm}$. La mayor concentración de restos arqueológicos se presenta entre los 10 y $50 \mathrm{~cm}$, reduciéndose drásticamente a partir de esta última profundidad. En la excavación no se distinguieron diferencias significativas en los sedimentos que contienen los materiales arqueológicos, resultando ser muy homogéneos, de composición limo-arenosa; hacia los últimos niveles $(60-80 \mathrm{~cm})$ los sedimentos comienzan a ser estériles arqueológicamente, de composición más arcillosa y de colores más rojizos.

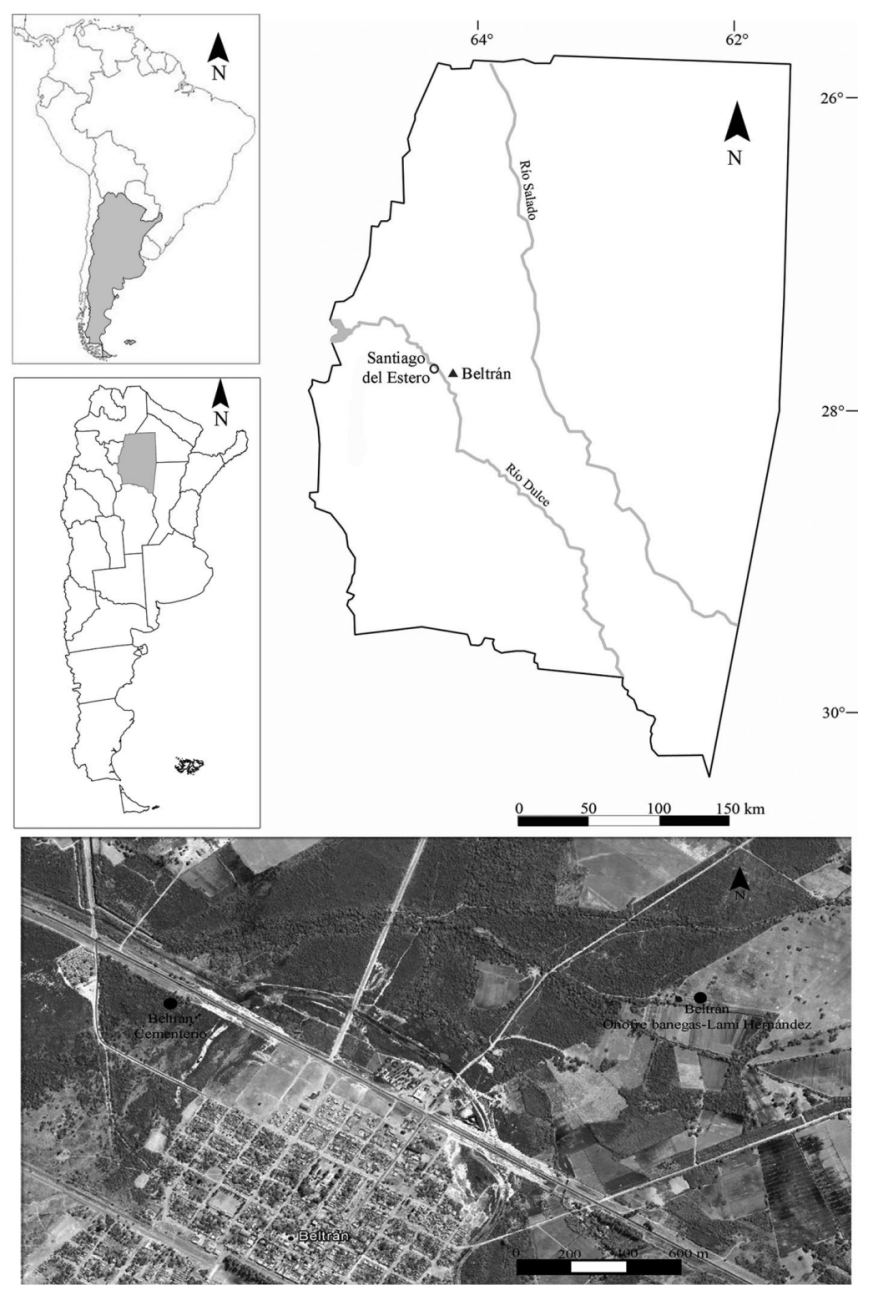

Figura 1. Ubicación del sitio Beltrán Onofre Banegas-Lami Hernández. 
El Período Tardío en la región Chaco-Santiagueña está caracterizado por la presencia de grupos portadores de la cerámica Sunchitúyoj y Averías (Togo 2004). En muchos sitios estas culturas se encuentran asociados, mientras que en otros se encuentran separados (Gramajo de Martínez Moreno 1978; Reichlen 1940; Togo 2004). La agrupación de Sunchitúyoj y Averías en la Tradición Cultural Chaco-Santiagueña por Lorandi (1978) se basa en el hecho de que sus diferencias fundamentales se centran en el tipo decorativo y estilístico de la cerámica, a que ambas presentan un patrón de asentamiento y un sistema económico con similares características, y sus diferencias principales serían de intensidad (e.g., mayor énfasis de las prácticas textiles en Averías y un aumento poblacional). Sin embargo, Togo (2004) considera útil mantener los nombres Sunchitúyoj y Averías en sentido amplio, ya que se encuentran sitios puros sin asociación entre estos, lo que denotaría la presencia de grupos humanos independientes dentro del territorio provincial que elaboraron materiales con tecnología y simbolismos diferentes, a pesar de la semejanza en cuanto al patrón de asentamiento (construcciones habitacionales sobre montículos naturales, artificiales o mixtos), economía (mixta, agrícola-ganadera y cazadora-recolectora), funebria y cierta tecnología cerámica (Togo 2004). Según nuevos fechados disponibles, los grupos Sunchitúyoj se desarrollaron entre el 1200 y $1500 \mathrm{dC}$ (Togo 2007) y podrían haber perdurado hasta la conquista española, por lo menos en algunas zonas de la provincia (Gramajo de Martínez Moreno 1978; Togo 2004). Por su parte, Averías se habría desarrollado muy cercano a la llegada de los conquistadores europeos, esto se desprende de las asociaciones directas con elementos hispánicos.

\section{Metodología}

Se analizaron los restos asignados a reptiles con el fin de identificar anatómica y taxonómicamente los restos. Dicha determinación se realizó mediante material de comparación depositado en la colección de la Cátedra de Anatomía Comparada de la Facultad de Ciencias Naturales y Museo de La Plata, y guías osteológicas (e.g., Krause 1978 a, 1978b). Se cuantificaron los materiales teniendo en cuenta las siguientes medidas de abundancia taxonómica y de partes esqueletarias: NISP (number of identified specimens per taxon, Payne 1975), MNI (minimum number of individuals; White 1953), MNE (minimum number of elements, Binford 1981; calculado por la frecuencia con que se hallan representadas una serie de zonas diagnósticas específicas de los elementos, Mengoni Goñalons 1999) y MAU\% (standardized minimum number of animal units, Binford 1984).

Se realizó el análisis contextual del registro durante la excavación, registrando cuevas o individuos en conexión orgánica que nos indique la muerte natural de los individuos del género Tupinambis en sus madrigueras, ya que suelen habitar cuevas durante los momentos de hibernación. También se observó en el análisis de laboratorio la presencia diferencial de pátinas de óxido de manganeso sobre las superficies articulares, lo que nos puede informar que los restos fueron enterrados articulados y que por procesos postdepositacionales, su posición original fue alterada. En este sentido, también se tuvieron en cuenta aquellos agentes que pudieron modificar la distribución del registro, como pueden ser los roedores y las raíces; para ello se estimó el porcentaje de especímenes con marcas generadas por estos agentes en el conjunto en general y en Tupinambis sp. en particular.

Se tuvieron en cuenta los estadios de meteorización para la muestra general (Behrensmeyer 1978, para mamíferos mayores a los $5 \mathrm{~kg}$ ) con el fin de interpretar relativamente la menor o mayor exposición de los restos, es decir, la menor o mayor chance de que los elementos sean desarticulados, destruidos, o que sea modificada la relación contextual del registro. 
Por otra parte se analizaron aquellas modificaciones óseas generadas por depredadores naturales como pueden ser aquellas generadas mecánicamente (e.g., mordisqueo) o por acción química (e.g., corrosión gástrica).

Se identificaron las modificaciones de la superficie ósea por factores antrópicos a nivel macroscópico y con lupa de mano de 15 aumentos. En las muestras analizadas, se tuvieron principalmente en cuenta las marcas de corte (Blumenschine et al. 1996; Mengoni Goñalons 1999), infiriendo la probable actividad que las produjo siguiendo los criterios de Binford (1981) y la termoalteración (Shipman et al. 1984; Stiner et al. 1995).

\section{Resultados}

En la muestra arqueofaunística predominan a través del NISP los peces, seguido por los mamíferos grandes (incluye a Lama sp.) -Tabla 1-. Entre los reptiles, se destacan los restos de Tupinambis sp. con el 5,25\% de la muestra general.

\begin{tabular}{|l|c|c|}
\hline Taxón & NTSP & NTSP\% \\
\hline Gastropoda & 78 & 1,38 \\
Teleostei & 1599 & 28,34 \\
Anura & 16 & 0,28 \\
Chelonoidis sp. & 37 & 0,66 \\
Ophidia & 43 & 0,76 \\
\hline Tupinambis sp. & 296 & 5,25 \\
\hline Aves indet." & 188 & 3,33 \\
Rhea americana* & 31 & 0,55 \\
Mammalia (grande= $>50 \mathrm{~kg}$ ) & 1047 & 18,55 \\
Lama sp. & 372 & 6,59 \\
Cervidae & 4 & 0,07 \\
Artiodactyla & 116 & 2,06 \\
Mammalia (mediano a grande*) & 417 & 7,39 \\
Mammalia (mediano= 15 a $50 \mathrm{~kg}$ ) & 628 & 11,13 \\
Mammalia (pequeño= 5 a $15 \mathrm{~kg}$ ) & 83 & 1,47 \\
Dasypodidae* & 207 & 3,67 \\
Carnivora & 5 & 0,09 \\
Lagostomus maximus & 119 & 2,11 \\
Dolichotinae & 49 & 0,87 \\
Mammalia (micromamíferos= $<1 \mathrm{~kg}$ ) & 308 & 5,46 \\
\hline Subtotat*** & 5643 & 100 \\
\hline
\end{tabular}

Tabla 1. Abundancia taxonómica. Referencias: *sin contar placas de la armadura ósea, ni cáscaras de huevo; ** Mammalia (mediano a grande) corresponde a aquellos especímenes que no pudieron ser asignados a ninguna de las categorías que lo componen, y establecidos a un tamaño cercano al de los $50 \mathrm{~kg} ;{ }^{* * *}$ estudio preliminar del sitio y sin tener en cuenta los indeterminados. 
Se recuperaron un total de 296 especímenes asignados a Tupinambis sp. y se calculó un MNI de 7 por la cantidad de maxilares (7 derechos) -Tabla 2-.

No se observó durante la excavación la presencia de restos articulados, y del análisis de la distribución diferencial de pátinas de óxido de manganeso, tampoco se infiere el enterramiento de elementos articulados de Tupinambis sp. (presencia de manchas extensivas que se producen en las distintas porciones de los elementos). En este sentido, consideramos que no se produjo la muerte natural de los individuos en sus madrigueras. Cabe mencionar que de otros animales de hábitos cavícolas se recuperaron individuos en conexión orgánica (i.e., Ctenomys sp.; Lagostomus maximus, Chaetophractus vellerosus), como así también se infiere el enterramiento de restos articulados de C. vellerosus por la distribución diferencial de pátinas de óxido de manganeso (trabajo en preparación). Por otra parte, se reconocieron en la excavación cuevas de animales, sin embargo, no se pudieron asociar elementos de Tupinambos sp. a dichas estructuras, y si a otros taxones (i.e., Ctenomys sp.; L. maximus, C. vellerosus).

El 77,78\% de los restos de mamíferos (> a $5 \mathrm{~kg}$ ) presentan estadios de meteorización bajos (estadios 0,1 y 2), el 20,35\% con estadio intermedio (estadio 3 ) y sólo el 1,86\% estadios altos (estadios 4 y 5) por lo que se infiere el relativamente rápido enterramiento de la mayoría de los materiales. Por otra parte, se observa en la muestra general que un 0,74 \% de los restos presentan marcas de roedores y el 2,51\% de raíces, siendo para Tupinambis sp. en particular, proporciones similares $(0,67 \%$ con marcas de roedores y $2,70 \%$ de raíces). $\mathrm{Si}$ bien estos agentes pudieron alterar la distribución espacial del registro, consideramos que los mismos no tuvieron un alto impacto en la muestra de Tupinambis sp., sobre todo si se tiene en cuenta que a través del análisis de la distribución diferencial de pátinas de óxido de manganeso no se observó el posible enterramiento de restos en estado articulado para este taxón.

Con respecto a los depredadores naturales, no se observaron evidencias de su actividad en los restos de Tupinambis sp., ya sea por acción mecánica (mordisqueo), ni por acción química (ausencia de corrosión gástrica).

Con respecto a la representación de partes esqueletarias, se observa la presencia de la mayoría de los elementos (Tabla 2), evidenciando la incorporación al registro de individuos enteros. A través del MAU\% (Tabla 2, Figura 2) se puede observar el predominio del cráneo y el radio, seguido por la mandíbula, escápula-coracoides, húmero, pelvis, fémur, tibia y peroné, una menor proporción de las vértebras, costillas, interclavícula y metapodios y nula para la ulna, elementos del basipodio y falanges.

Entre las evidencias de acumulación antrópica, se recuperaron tres elementos craneanos con marcas de corte (1,01\% de los especímenes de Tupinambis sp.). En este caso se infiere la desarticulación de la mandíbula del resto del cráneo por observarse marcas de corte sobre los elementos que unen dichas estructuras. Las marcas se registraron en un articular (elemento de la mandíbula) y sobre dos cuadrados (elementos donde articula la mandíbula), las mismas se presentan sobre las carillas articulares de los elementos (Figura 3). 


\begin{tabular}{|c|c|c|c|c|c|c|}
\hline Región & Parte esqueletaria & NISP & MNI & MNE & MAU & MAU $\%$ \\
\hline \multirow{18}{*}{ Cráneo } & Supraoccipital & 1 & 1 & 1 & 1 & 20 \\
\hline & Otoccipital & 2 & 1 & 2 & 1 & 20 \\
\hline & Basioccipital & 2 & 2 & 2 & 2 & 40 \\
\hline & Parietal & 3 & 1 & 2 & 1 & 20 \\
\hline & Prefrontal & 4 & 2 & 4 & 2 & 40 \\
\hline & Frontal & 3 & 3 & 3 & 3 & 60 \\
\hline & Escamoso & 3 & 2 & 3 & 1,5 & 30 \\
\hline & Yugal & 7 & 4 & 7 & 3,5 & 70 \\
\hline & Cuadrado & 8 & 4 & 8 & 4 & 80 \\
\hline & Postorbital & 5 & 3 & 5 & 2,5 & 50 \\
\hline & Pterigoides & 7 & 4 & 7 & 2,5 & 50 \\
\hline & Ectopterigoides & 5 & 3 & 5 & 2,5 & 50 \\
\hline & Proótico & 2 & 1 & 2 & 1 & 20 \\
\hline & Parabasiesfenoides & 3 & 3 & 3 & 3 & 60 \\
\hline & Nasal & 3 & 2 & 3 & 1,5 & 30 \\
\hline & Palatino & 4 & 2 & 4 & 2 & 40 \\
\hline & Premaxilar & 3 & 3 & 3 & 3 & 60 \\
\hline & Maxilar & 10 & 7 & 10 & 5 & 100 \\
\hline \multirow[t]{6}{*}{ Mandibula } & Articular & 5 & 4 & 5 & 2,5 & 50 \\
\hline & Angular & 5 & 3 & 5 & 2,5 & 50 \\
\hline & Suprangular & 1 & 1 & 1 & 0,5 & 10 \\
\hline & Dentario & 5 & 4 & 5 & 2,5 & 50 \\
\hline & Coronoides & 2 & 1 & 2 & 1 & 20 \\
\hline & Esplenial & 2 & 1 & 2 & 1 & 20 \\
\hline Columna vertebral & Vértebras & 128 & 2 & 128 & 1,66 & 33,2 \\
\hline Costillas & Costillas & 18 & 1 & 18 & 0,56 & 11,2 \\
\hline \multirow[t]{4}{*}{ Miembro anterior* } & Escápula-coracoides & 5 & 4 & 5 & 2,5 & 50 \\
\hline & Interclavicula & 1 & 1 & 1 & 1 & 20 \\
\hline & Húmero & 8 & 3 & 6 & 3 & 60 \\
\hline & Radio & 8 & 4 & 8 & 4 & 80 \\
\hline \multirow[t]{6}{*}{ Miembro posterior ${ }^{*}$} & Ileon & 6 & 6 & 6 & 3 & 60 \\
\hline & Isquion & 4 & 3 & 4 & 2 & 40 \\
\hline & Pubis & 4 & 2 & 4 & 2 & 40 \\
\hline & Fémur & 6 & 3 & 4 & 2 & 40 \\
\hline & Tibia & 8 & 3 & 5 & 2,5 & 50 \\
\hline & Peroné & 4 & 2 & 4 & 2 & 40 \\
\hline \multirow[t]{2}{*}{ Elementos distales } & Metapodios & 1 & 1 & 1 & 0,05 & 1 \\
\hline & Total & 296 & 7 & 288 & - & - \\
\hline
\end{tabular}

Tabla 2. Abundancia taxonómica de Tupinambis sp. (NISP y MNI) y representación de partes (MNE, MAU y MAU\%). *incluye a las cinturas 


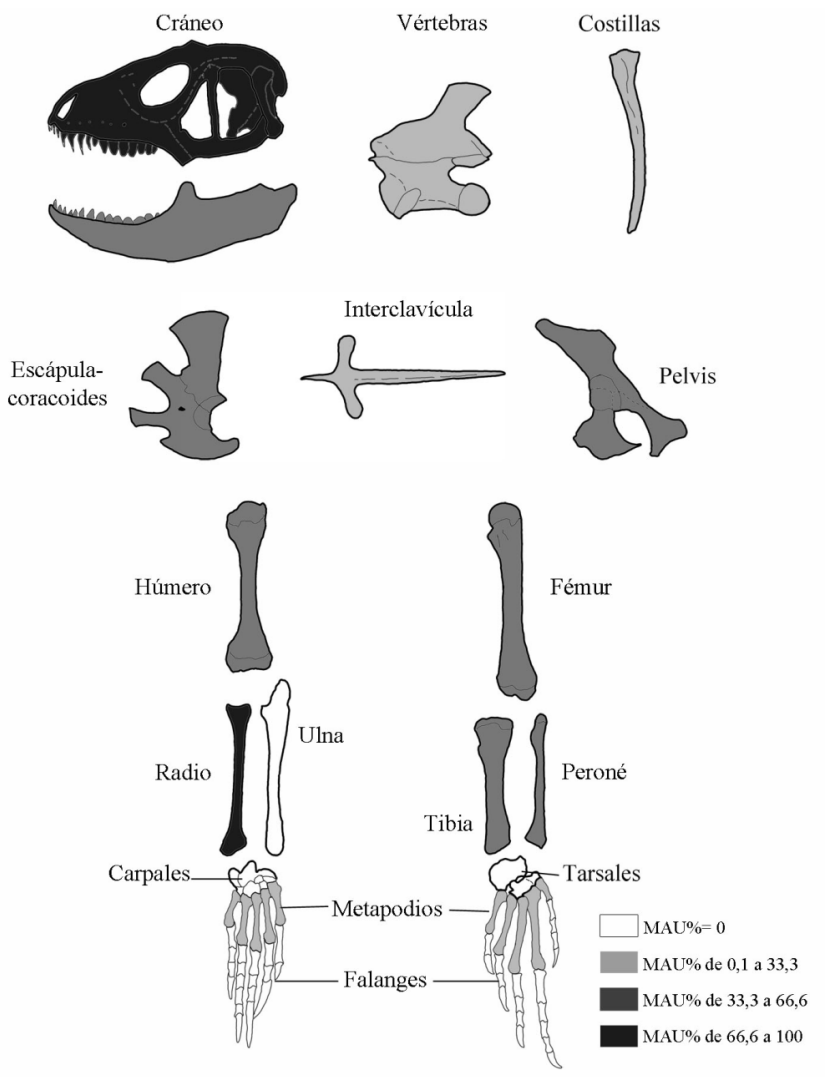

Figura 2. Representación de partes esqueletarias. MAU\%



Figura 3. Marcas de corte en especímenes de Tupinambis sp. a- Cuadrado, vista ventral; b-Articular, vista oclusal y posterior. 

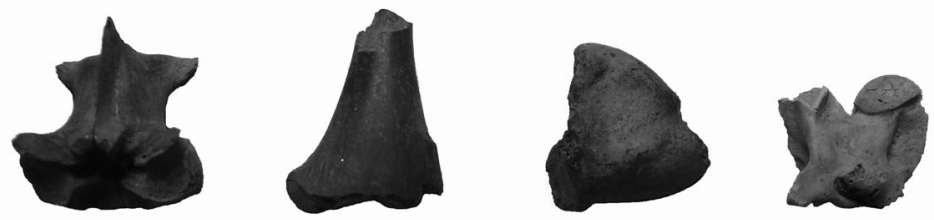

$1 \mathrm{~cm}$

Figura 4- Elementos termoalterados. De izquierda a derecha: vértebra, porción distal de húmero y epífisis proximal de tibia carbonizados, y fragmento de vértebra calcinado.

Con respecto a la termoalteración, el 5,40\% de los especímenes de Tupinambis sp. presenta esta característica, y de este porcentaje, el 6,25\% se encuentra quemado ${ }^{1}$ (un pterigoides), el 56,55\% carbonizado (un premaxilar, cinco vértebras, dos fragmentos distales de húmero y una epífisis proximal de tibia), y el 37,5\% calcinado (cuatro vértebras, una costilla y una fragmento distal de tibia) -Figura 4-. En este sitio predominan las vértebras con evidencias de termoalteración, seguido por los elementos de los miembros y en menor medida del cráneo.

\section{Discusión y conclusiones}

A través del análisis contextual de los restos, así como de las cuevas registradas durante la excavación, y el posterior análisis en el laboratorio de la distribución diferencial de pátinas de óxido de manganeso sobre los especímenes, se considera que las evidencias no concuerdan con la muerte natural de los individuos de Tupinambis sp. en sus madrigueras. Por otra parte, el análisis de la meteorización, las modificaciones generadas por raíces y los roedores en la muestra general nos informa que el registro no fue alterado significativamente por estos agentes. Hay que tener en cuenta que la distribución espacial se vio alterada por la construcción de cuevas de animales de hábitos cavícolas, sin embargo, los restos de Tupinambis sp. no se encontraron vinculados a las mismas.

Descartando así la muerte natural de los individuos en el registro, cabe mencionar que tampoco se observaron evidencias de la incorporación por depredadores naturales, y por contrapartida se observaron marcas de corte y termoalteración, infiriendo la actividad humana como el principal generador del registro de Tupinambis sp. Asimismo, la representación de partes de este lagarto coincide con la incorporación de las presas en forma entera a los lugares de consumo. La diferencia en la proporción en que se recuperaron los elementos puede deberse a cuestiones de procesamiento como de sesgos en la recuperación e identificación (principalmente de aquellos restos pequeños como los elementos del basipodio y acropodio).

Cabe mencionar que los restos de Tupinambis sp. son recurrentes en los sitios arqueológicos de la región Chaco-Santiagueña (del Papa 2012), si bien en menor proporción que en el sitio aquí estudiado. En este sentido se han observado marcas de corte tanto en el sitio Villa la Punta (en un húmero) y en el sitio Beltrán Cementerio (en una mandíbula), este último muy próximo a Beltrán Onofre Banegas- Lami Hernández -Figura 1-(del Papa 2012). A su vez, en otras regiones del país (Sierras Centrales, Chaco Meridional y Pampeana) hay una preponderancia de marcas de corte sobre elementos de las mandíbulas (Medina 2008; Quintana et al. 2002; Santini 2009), coincidente con lo observado en el sitio aquí estudiado. 
Por otra parte, en el sitio Beltrán Onofre Banegas-Lami Hernández se han recuperado restos termoalterados de Tupinambis sp. que en general presentan signos de combustión intensa (entre carbonizados y calcinados), y si bien algunos elementos podrían corresponder con el modelo de cocción sugerido por Medina y Teta (2010) para roedores caviomorfos (termoalteración en los elementos distales de los miembros), otros escapan a dicho modelo, como son las vértebras, costillas y elementos del cráneo, por lo que se interpreta que estos restos pudieron ser descartados en estructuras de combustión. Estas evidencias concuerdan con las observadas en otros sitios de la región del contexto agroalfarero para este taxón, como los sitios Villa la Punta, Maquijata, La Cañada y Media Flor (del Papa 2012) y para la mayoría de los taxones de los sitios analizados para este período (del Papa 2012).

El ingreso de individuos completos a los lugares de consumo podría estar evidenciando el uso integral de este recurso. En este sentido, la región de la cola (representada por vértebras caudales) sería la porción con más aporte de carne, como lo mencionan los pobladores rurales actuales de la provincia (Leon y Togo 2013); por otro lado podrían haber utilizado el cuero, que si bien no hay evidencias de su extracción en este sitio, se ha comprobado dicha acción para el sitio Beltrán Cementerio (del Papa 2012; del Papa et al. 2013), muy próximo al sitio aquí analizado y de mismo contexto cultural.

Hay que destacar que actualmente Tupinambis sp. es muy importante simbólica y medicinalmente para los pobladores campesinos de Santiago del Estero, Tobas y Wichís del Chaco central y otras zonas de la Argentina (Arenas 2003; Basualdo et al. 1985; Quintana et al. 2004). Sin embargo, por el momento es muy difícil poder contrastar la importancia simbólica de este saurio con el registro. Si bien Tupinambis sp. en la muestra no supera el $6 \%$ del NISP total, los mismos representan un recurso complementario y de importancia en relación al uso estacional de las presas.

Agradecimientos: A Valeria Accinelli por la traducción del resumen al inglés. Al profesor Juan Carlos Cejas por facilitar el trabajo de campo y su constante apoyo a los trabajos arqueológicos de la zona, a doña Elda y al Sr. Lami Hernández por permitirnos trabajar en sus propiedades y facilitar sus instalaciones, al ex intendente de Beltrán Miguel Álvarez por el apoyo brindado. A los evaluadores anónimos cuyos comentarios ayudaron a mejorar la confección del trabajo. Lo aquí expresado es responsabilidad de los autores. Este trabajo forma parte del proyecto de beca Postdoctoral del CONICET de uno de los autores, y cuenta con financiamiento del proyecto del Programa de Incentivos para Docentes-Investigadores, Código: 11/N601. Facultad de Ciencias Naturales y Museo, UNLP, director: Dr. Luciano De Santis.

\section{Notas}

1 Huesos quemados: aquellos que presentan un bajo grado de termoalteración, de color marrón oscuro a negro y que por lo general se presentan en una porción del elemento.

\section{Bibliografía citada}

Arenas, P.

2003 Etnografía y Alimentación Entre los Tobas Ñachilamoleek y Wichi-Lhukutas del Chaco Central (Argentina). Pastor Arenas editor, Buenos Aires. 
Basualdo, M.A.; J. Togo y N. Urtubey

1985 Aprovechamiento socioeconómico de la fauna autóctona de Santiago del Estero (inventario y uso popular más frecuente). Indoamérica 1. Publicación del Laboratorio de Antropología, Facultad de Humanidades, Universidad Nacional de santiago del Estero, Santiago del Estero.

Behrensmeyer, A. F.

1978 Taphonomic and ecologic information from bone weathering. Paleobiology 4: 150-162.

Binford, L.R.

1981 Bones: Ancient Men and Modern Myths. Academic Press, New York.

Binford, L.R.

1984 Faunal Remains from Klasies River Mouth. Academic Press, Orlando.

Blumenschine, R. J.; C. W. Marean y S. D. Capaldo

1996 Blind test of inter-analyst correspondence and accuracy in the identification of cut marks, percussion marks, and carnivore tooth marks on bone surfaces. Journal of Archaeological Science 23: 493-507.

Brizuela, S.

2010 Los lagartos continentales fósiles de la Argentina (excepto Iguania). Tesis Doctoral. Facultad de Ciencias Naturales y Museo, UNLP, La Plata.

del Papa, L.M.

2012 Una aproximación al estudio de los sistemas de subsistencias a través del análisis arqueofaunístico en un sector de la cuenca del Río Dulce y cercanías a la Sierra de Guasayán. Tesis Doctoral. Facultad de Ciencias Naturales y Museo, UNLP, La Plata.

del Papa, L.M., L.J.M. De Santis y J. Togo

2013 Zooarqueología del sitio Beltrán Cementerio, Santiago del Estero. Cuadernos del Instituto Nacional de Antropología y Pensamiento Latinoamericano. Series Especiales 1: 168-180

Fitzgerald, L. A.

1992 La historia natural de Tupinambis. Revista de la Universidad Nacional de Asunción 3: 71-72.

Gramajo de Martínez Moreno, A.

1978 Evolución cultural en el territorio santiagueño a través de la arqueología. Serie Monográfica № 5. Publicación del Museo Arqueológico "Emilio Y Duncan Wagner", Santiago del Estero.

Krause, L.

1978 a Osteologia pós-craniana de Tupinambis teguixin (L., 1758) sensu Boulenger, 1885. Parte I: esqueleto axial (vértebras e costelas). (Lacertilia, Scincomorpha, Teiidae). Revista Brasileira de Biologia 38 (2): 481-499.

Krause, L.

1978 b Osteologia pós-craniana de Tupinambis teguixin (L., 1758) sensu Boulenger, 1885. Parte II: esqueleto apendicular (cintura e membros). (Lacertilia, Scincomorpha, Teiidae). Revista Brasileira de Biologia 38 (3): 509-521. 
Ledesma, N.R.

1979 La verdad sobre el clima de Santiago del Estero. Cuaderno de Cultura 10 (17), Municipalidad de Santiago del Estero, Santiago del Estero.

Leon, D.C. y J. Togo

2013 La utilización de la fauna por pobladores rurales santiagueños. Implicancias arqueológicas. Trabajo presentado en el XVIII Congreso Nacional de Arqueología Argentina. La Rioja

Lorandi, A.M.

1978 El Desarrollo Cultural Prehispánico en Santiago del Estero. Argentina. Journal de la Société des Américanistes 65 (1): 63-85.

Medina, M.E.

2008 Diversificación económica y uso del espacio en el Tardío Prehispánico del norte del Valle de Punilla, Pampa de Olaen y Llanura Noroccidental (Córdoba, Argentina). Tesis Doctoral. Facultad de Filosofía y Letras. UBA, Buenos Aires.

Medina, M.E. y P. Teta

2010 Alteración térmica y consumo de roedores caviomorfos en Quebrada del Real 1 (Pampa de Achala, Córdoba): una aproximación experimental. Trabajo presentado en el XVII Congreso Nacional de Arqueología Argentina, Mendoza.

Mengoni Goñalons, G.L.

1999 Cazadores de guanacos de la estepa patagónica. Sociedad Argentina Antropología, Colección tesis doctorales, Buenos Aires.

Payne, S.

1975 Partial recovery and sample bias. Archaeozoological Studies (ed. Por A. T. Clason), pp. 7-17. North Holland, Amsterdam.

Porini, G.M.

2006 Proyecto Tupinambis. Una propuesta para el manejo de Tupinambis rufescens y T. merianae en la Argentina. Manejo de Fauna Silvestre en la Argentina. Programas de Uso Sustentable (ed. por M. L. Bolkovic y D. Ramadori), pp. 65-75. Dirección de Fauna Silvestre, Secretaría de Ambiente y Desarrollo Sustentable, Buenos Aires.

Quintana, C.A.; F. Valverde y D.L. Mazzanti

2002 Roedores y lagartos como emergentes de la diversificación de la subsistencia durante el Holoceno Tardío en sierras de la región Pampeana Argentina. Latin American Antiquity 13 (4): 455-473.

Quintana, C.A.; D. Mazzanti y F. Valverde

2004 El lagarto overo como recurso faunístico durante el Holoceno de las sierras de Tandilia Oriental, provincia de Buenos Aires. La Región Pampeana, su pasado arqueológico (ed. por C. Gradín y F. Oliva), pp. 347-353. Editorial Laborde, Buenos Aires.

Reichlen, $\mathrm{H}$.

1940 Reserches Archeologiques dans la province de Santiago del Estero (Rep. Argentine). Journal de la Société des Americanistes 32 : 133-225. 
Richard, E.

1999 Tortugas de las regiones áridas de Argentina: Contribución al conocimiento de las tortugas de las regiones áridas de Argentina (Chelidae y Testudinidae) con especial referencia a los aspectos ecoetológicos, comerciales y antropológicos de las especies del complejo chilensis (Chelonoidis chilensis y C. donosobarrosi) en la provincia de Mendoza. L.O.L.A. Literature of Latin America, Buenos Aires.

\section{Santini, M.}

2009 Prehistoria de la región meridional del Gran Chaco. Aportes del análisis de restos faunísticos en la reconstrucción de las estrategias adaptativas de los grupos aborígenes. Tesis Doctoral. Facultad de Ciencias Naturales y Museo, UNLP, La Plata.

Shipman, P.; G.F. Foster y M. Schoeninger

1984 Burnt bones and teeth: an experimental study of colour, morphology, crystal structure and shrinkage. Journal of Archaeological Science 11: 307-325.

Stiner, M.C.; S.L. Kuhn; S. Weiner y O. Bar-Yosef

1995 Differential burning, recrystalization, and fragmentation of archaeological bone. Journal of Archaeological Science 22: 223-237.

Togo, J.

2004 Arqueología Santiagueña: Estado actual del Conocimiento y Evaluación de un Sector de la Cuenca del Río Dulce. Tesis Doctoral. Facultad de Ciencias Naturales y Museo, UNLP, La Plata.

Togo, J.

2007 Los fechados radiocarbónicos de Santiago del Estero. Actas de resúmenes ampliados del XVI Congreso de Arqueología Argentina, Tomo 3: 227-232. San Salvador de Jujuy.

White, T.E.

1953. A method of calculating the dietary percentage of various foods animals utilized by various aboriginal peoples. American Antiquity 18 (4): 396-398. 